\title{
Cortactin (CTTN) overexpression in osteosarcoma correlates with advanced stage and reduced survival
}

Cecilia Folio ${ }^{\mathrm{a}}$, Marta Zalacain ${ }^{\mathrm{a}}$, Carolina Zandueta $^{\mathrm{b}}$, Cristina Ormazábal ${ }^{\mathrm{b}}$, Luis Sierrasesúmaga ${ }^{a}$, Mikel San Julián ${ }^{\mathrm{c}}$, Javier de las Rivas ${ }^{\mathrm{d}}$, Gemma Toledo $^{\mathrm{e}}$, Fernando Lecanda ${ }^{\mathrm{b}}$ and Ana Patiño-García ${ }^{\mathrm{a}, *}$

${ }^{a}$ Laboratory of Pediatrics, University Clinic of Navarra, Pamplona, Spain

${ }^{\mathrm{b}}$ Division of Oncology, Adhesion and Metastasis Laboratory, Center for Applied Biomedical Research (CIMA), University of Navarra, Pamplona, Spain

${ }^{\mathrm{c}}$ Department of Orthopedics, University Clinic of Navarra, Pamplona, Spain

${ }^{\mathrm{d} B}$ Bioinformatics and Functional Genomics Research Group, Cancer Research Center (IBMCC-CIC), CSIC and University of Salamanca, Salamanca, Spain

${ }^{\mathrm{e}}$ Department of Pathology, MD Anderson Cancer Center, Madrid, Spain

*Corresponding author: Ana Patiño-García Ph.D.

Laboratory of Pediatrics, University of Navarra, Pamplona 31080. Navarra, Spain.

Tel.: +34 948 425600; Fax: +34 948425649 E-mail: apatigar@ unav.es.

\section{ABSTRACT.}

Background. The cortactin (CTTN) gene has been found, by transcriptomic profiling, to be overexpressed in pediatric osteosarcoma. The location of CTTN at 11q13 and the role of cortactin in cytoskeleton restructuring make CTTN of interest as a potential biomarker for osteosarcoma.

Materials and methods. Osteoblasts were isolated from 20 high-grade osteosarcomas before chemotherapy, and paired with cell samples from normal tissue, prior to RNA expression analysis on HG-U133A chips (Affymetrix). Semiquantitative CTTN mRNA expression was analyzed by real-time PCR. An osteosarcoma tissue microarray (TMA) containing 233 tissue spots from 48 patients was used for an immunohistochemical (IHC) study of cortactin.

Results. Transcriptomic profiling and real-time PCR analysis indicated increased CTTN expression in osteosarcomas $(p=0.001$, Student's T test). TMA IHC showed cortactin to be present more frequently and in greater abundance in osteosarcomas than non-tumoral osteoblastic samples ( $p<0.006$, Mann-Withney test). Analysis of clinical outcomes indicated that overall survival for patients with primary tumors positive for cortactin was significantly lower than that for patients with cortactin negative (or only weakly staining) tumors $(\mathrm{p}=0.0278$, Log-rank test $)$.

Conclusions. Our preliminary data support the hypothesis that over-expression of cortactin, contained in the 11q13 amplicon, is involved in osteosarcoma carcinogenesis. The potential of cortactin overexpression as a biomarker for osteosarcoma is consolidated.

Keywords. Cortactin, expression analysis, metastasis, osteosarcoma, survival 


\section{INTRODUCTION}

Osteosarcoma is the most common primary malignant tumor of bone, characterized by production of malignant osteoid and a high propensity to metastasize [1]. It most frequently occurs in the second decade of life. Several factors have been related to survival, including the presence of metastatic disease and the histologic response to preoperative treatment $[2,3]$. Despite efforts in new therapeutic modalities based on neoadjuvant chemotherapy followed by surgical resection and postoperative chemotherapy, overall survival rates rarely exceed $60-65 \%$ [2].

We have previously reported the transcriptomic profiling [4] of pediatric osteosarcoma based on a paired analysis of tumoral and normal cell lines derived from high-grade conventional osteosarcomas. This approach enabled assessment of the changes associated with oncogenesis and determination of molecular alterations influencing differential clinical behaviour and/or response to treatment. We found a robust clustering that revealed a novel subset of genes associated with the carcinogenesis of pediatric osteosarcoma, in which the transcription factor EBF2 was found to be highly expressed in tumor samples.

In the study reported here, CTTN was one of the most significant genes overexpressedin osteosarcomas. The product of the CTTN gene (previously known as EMS1) is cortactin, an actin filament-binding protein and target of various kinases, which has emerged as a central element connecting signalling pathways with cytoskeleton restructuring. Remodelling of the actin cytoskeleton has effects on cell migration, motility, and adhesion, as well as on tumor invasion and metastasis [5]. Here we report the identification and validation of cortactin (and overexpression of CTTN) as a potential new marker for poor outcome in osteosarcoma.

A large body of evidence implicates cortactin in the pathogenesis of human neoplasia. Most frequently, CTTN overexpression occurs through chromosomal amplification of the 11q13.3 region [6], a genetic event that has been described in osteosarcoma [7]. In addition to cortactin, a number of other genes potentially involved in carcinogenesis are present in the 11q13 amplicon, including the CCDN1 gene, which encodes cyclin D1. CTTN and cyclin D1 have been considered the most likely promotors of tumor aggressiveness since, unlike many of the other genes in the amplicon, they are consistently overexpressed upon amplification [6].

\section{MATERIALS AND METHODS}

\subsection{Patients and clinical samples}

Primary tumor samples were obtained by needle biopsy from 20 high-grade osteosarcomas before induction chemotherapy, and paired-normal osteoblasts were isolated when patients underwent surgery (Table 1). All patients were treated at the Pediatric Oncology Unit of the University Clinic of Navarra. All samples were obtained with written informed consent from patients and/or their parents, and the protocol was approved by the local ethics committee (CEIC-CUN). Human osteoblasts were isolated and characterized according to procedures we have published previously $[4,8]$. Normal 
osteoblastic cells were passaged 1 to 5 times, whereas tumoral cells were passaged between 20 and 40 times; in all cases, cells were 90\% confluent and in logarithmic growth phase prior to molecular analysis.

Cell lines were designated by the number of the patient followed by B (biopsy, i.e. tumor-derived lines) or N (normal, i.e. paired normal cell lines).

\subsection{RNA extraction for microarray analysis.}

RNA was isolated, labelled and hybridized on HGU133A chips (Affymetrix) by Progenika Biopharma as previously described [4]. The test set comprised 14 samples: osteosarcoma cell lines and the paired osteoblastic control cell lines derived from 7 patients (numbers 473, 475, 486, 491, 500, 524 and 531 in Table 1).

\subsection{Gene expression validation by real-time PCR}

To validate CTTN gene expression results, we performed real-time PCR assay by means of TaqMan Micro Fluidic Cards on the tumour-derived cells from patients 554, $588,595,598,605,628,631,636,648,652,661,664$ and 669 (Table 1). Total RNA was isolated from confluent cultures using Trizol RNA $(2 \mu \mathrm{g})$. After treatment with DNase I, RNA was reverse transcribed using SuperScript II reverse transcriptase and random primers.

Semiquantitative analysis of CTTN mRNA expression in the tumour samples was carried out by real-time polymerase chain reaction (RT-PCR) with the ABI PRISM 7300 Sequence Detector and the software Sequence Detector version 1.4 (PerkinElmer/Applied Biosystems, Foster City, California). The semiquantitative CTTN mRNA level was expressed as a percentage relative to that of glyceraldehyde-3phosphate dehydrogenase mRNA (GAPDH), which was determined with TaqMan assays (Hs00193322 ml, Applied Biosystems). Relative levels of expression were determined by the $2^{-\overline{\Delta C \mathrm{Ct}}}$ method, and every assay was performed in triplicate.

\subsection{Immunohistochemistry}

An osteosarcoma tissue microarray (TMA) containing 233 tissue spots derived from 48 patients was developed in our institution using samples from our archives. Tissues in the microarray included normal bone $(17.2 \%)$, osteosarcomas at diagnosis $(26.3 \%)$ and after induction with chemotherapy $(11.1 \%)$, relapsed tumors $(10.1 \%)$, and metastatic tumors $(35.4 \%)$. Tissue sections were formalin-fixed and paraffin-embedded. Paraffin was removed from the tissues and the sections were hydrated through a graded series of ethanol solutions. Endogenous peroxidase activity was quenched with 3\% hydrogen peroxidase for $10 \mathrm{~min}$. Microwave antigen retrieval was carried out with EDTA $(0.5$ $\mathrm{mmol} / \mathrm{L}, \mathrm{pH} 8$ ) for $2 \times 15 \mathrm{~min}$. Nonspecific binding sites were blocked with $5 \%$ goat normal serum in TBS-Tween 20 (with DAKO washing buffer) for $30 \mathrm{~min}$. Sections were incubated with anti-cortactin antibody (\#ab81208, Abcam, Cambridge, UK) overnight at $4{ }^{\circ} \mathrm{C}$. The working dilution was 1:300. After rinsing with TBS, sections were incubated with polyclonal Envision complex (DAKO). Peroxidase activity was 
shown by 3,3 '-diaminobenzidine. Finally, sections were washed in water, lightly counterstained with hematoxylin, dehydrated, and mounted in DPX.

For the TMA, a semiquantification procedure was performed according to a score that took into account the extension and intensity of the immunoreactivitysignal. The " 0 " score was assigned when the sample was negative (i.e. less than $10 \%$ of the cells were positive). Score "1" indicates a weak signal; Score "2", a strong and widespread signal; and Score "3", an intense and extensive [9].

\subsection{Statistical analysis}

Because this study was designed as a confirmatory analysis focused on specific genes that were previously shown to have statistically significant expression changes in our set of osteosarcomas, multiple test correction was not applicable. Gene expression levels were compared with the Mann-Whitney $U$ test or the Kruskal-Wallis test. Differences in survival were plotted in Kaplan-Meier curves and were tested for statistical significance with the Log-rank test (Mantel Cox correlation). The statistical analysis was done with the SPSS (Statistical Package for the Social Sciences, version 15.0) software. We used $p<0.05$ as criterion of statistical significance.

\section{RESULTS}

The application of transcriptomic profiling and stringent bioinformatic processing to a set of 7 tumourderived in vitro cultures from chemonaive osteosarcoma samples paired with 7 normal osteoblastic cell populations identified CTTN as one of several genes differentially upregulated $(\mathrm{p}=0.038)$ in the osteosarcoma samples. Real-time PCR assay with Micro Fluidic Cards validated this finding $(\mathrm{p}=0.003)$. Expression levels in tumoral tissues was additionally tested by real-time PCR in an independent subset of 13 tumourderived and 12 normal osteoblastic cell lines derived from primary tissues; there was a significant increase of CTTN mRNA in tumors $(\mathrm{p}=0.001$, Student's T test, Fig. $1)$.

On establishing the above result, and in view of the fact that previous studies have found both CTTN and CCND1 to be overexpressed in some tumours with the 11q13 amplification, we retrospectively checked the expression of U133A GeneChip probesets 208711_s at and 208712_at, both of which concern sequences located within the CCND1 gene. At the stringency of the statistical test used in the cDNA array analysis, there were no significant differences in the expression of these sequences.

The immunohistochemical (IHC) analysis (based on a monoclonal antibody to cortactin that was used on a tissue microarray that included 48 osteosarcoma patients) showed significantly higher expression of cortactin in osteosarcoma tissue compared to nontumoral osteoblastic tissue from normal bone $(p<0.006$, Mann-Withney test). We found a significantly higher number of positive cases, and the intensity of immunoreactivity was higher, in metastasis and relapse samples as compared with samples from primary tumors $(\mathrm{p}=0.028$, Kruskal-Wallis test) (Figs 2 and 3). Of samples from primary tumors and lung metastases, $25 \%$ were positive, and the pattern of staining was typically patchy and light. In cases of local recurrences and distant bone 
metastases, however, all samples were positive, and staining was generally uniform and dense.

Analysis of outcomes after stratification of patients according to IHC scores indicated that overall survival for patients whose primary tumors were positive for cortactin (IHC scores higher than $2, \mathrm{n}=20$ ) was significantly reduced relative to that for patients with weak cortactin staining (IHC scores lower or equal to $2, \mathrm{n}=8, \mathrm{p}=0.0278$, Log-rank test, Fig. 4). The association between survival and the cortactin IHC was not maintained after correction for multiple testing, with only a tendency for association remaining (adjusted $\mathrm{p}$-value $=0.101)$, probably due to the small sample size $(\mathrm{n}=28)$.

In conjunction, the results of the various areas of the study suggest an inverse relationship between patient survival and amounts of cortactin in tumor cells.

\subsection{DISCUSSION}

Cortactin has been described as an actin-associated scaffolding protein, which binds and activates the actinrelated protein $2 / 3$ complex, and has emerged as a central element connecting signalling pathways with cytoskeleton restructuring [10,11]. Remodelling of the actin cytoskeleton has effects on cell migration, motility, and adhesion, as well as on tumor invasion and metastasis [5].

Cortactin is overexpressed in many types of human cancers, including head and neck and esophageal squamous carcinomas and colorectal, gastric, hepatocellular, breast and ovarian cancers $[11,12]$.

The gene coding for cortactin is in the chromosome region 11q13 and is amplified in a wide variety of human cancers, but scarcely in osteosarcoma [13], suggesting that the 11 q13 amplicon may harbor key gene(s) involved in carcinogenesis regardless of tissue types [14]. Amplification of 11q13 is specific for certain malignancies and seems to occur at high frequency in some adult carcinomas (breast, head and neck, ovary, squamous cell carcinoma, melanoma and hepatocellular carcinoma). Within these patients, the amplification dentifies a subgroup of patients with poor prognosis (see [6] for a review).

Several oncogenes and cancer-related genes, such as CCND1, FGF4, FGF3, CTTN, and PAK1, have been mapped to the $11 \mathrm{q} 13$ chromosome region. The amplification or involvement of these genes in osteosarcoma has not been reported so far. CCND1 and CTTN are coamplified, at the same level, in most adult carcinomas with 11q13 amplification, which may indicate that both genes confer selective advantage to these tumors [6].

The relationship between cortactin expression, CTTN over-expression and the clinicopathological parameters of pediatric osteosarcoma remains largely unexplored. Our findings indicate that marked cortactin IHC positivity associates with poor survival. In some studies immunostaining for cyclin D1 was weak or absent in most tumor samples and cyclin D1 overexpression was not detected $[15,16]$. The implication is that the gene overexpressed in the 11q13 amplicon in osteosarcoma might be CTTN, at least in a subset of tumors. Also, it has been reported that there is overexpression of the genes 
in the amplified region without amplification, suggesting that there must be mechanisms other than amplification to activate the biologially important genes in that region. In the specific case of osteosarcoma, the alteration of the G1 phase of the cell cycle is a frequent event, by the abrogation of either the Rb1 or the TP53 pathways. Our data is congruent with reports that cyclin D1 is usually expressed at a low level in osteosarcoma, a finding that supports the idea of there being mechanisms other than amplification involved in activation of genes within the amplicon region [17].

As reported before, various studies have found an association between 11q13 amplification and poor prognosis, including higher pathological stage, lymph node and distant metastasis, and decreased survival [18]. The consistent over-expression of CTTN in 11q13-amplified tumors along with its ubiquitous presence in cell motility structures, such as lamellipodia and invadopodia, have generated a great deal of interest in the role of cortactin in tumor invasion.

On the basis of the localization and function of cortactin in non-transformed cell lines, it was suggested that over-expression of cortactin in human cancers might lead to increased cell migration [11,12]. Cell migration is required for many biological processes, and the aberrant regulation of cell migration drives progression of many diseases, including cancer invasion and metastasis. Therefore, understanding the fundamental mechanisms of cell migration is critical for our understanding of both basic biology and the pathology of disease [19]. In view of this model of cancer, we not only analyzed the expression of CTTN in paired normal and tumoral tissues and of cortactin in different stages of the disease (normal bones, primary osteosarcomas, metastases and local relapses) but also compared the IHC expression of cortactin in primary osteosarcoma tissues from metastatic and non-metastatic patients. As reported here, both analyses indicated that, also in osteosarcoma, cortactin could be associated with the development of metastatic disease and therefore reduced survival.

In conclusion, our data suggest an involvement of the CTTN gene in the carcinogenesis of pediatric osteosarcoma, and that over-expression of this gene and the corresponding marked IHC positivity for the protein cortactin could be predictors of metastasis and reduced survival for pediatric patients with this tumor. Nevertheless, the number of cases analyzed in this paper does not warrant that our results be regarded without some degree of caution. In addition, further studies should be performed to confirm the specific mechanism/s by which CTTN and CCND1 are overexpressed and whether there are other genes within or in the proximity of the 11q13 amplicon that are also involved in osteosarcoma.

\section{ACKNOWLEDGEMENTS}

To all patients and families. We are indebted to David Burdon for the critical reading of this manuscript.

This work was supported by a grant from the Spanish Ministry of Science and Technology, FIS, ISCIII, ref. PI10/01580 and by a grant from CAN, "Tú eliges, tú decides" (ref. 12488). 


\section{REFERENCES}

1) A.K. Raymond, A.G. Ayala and S. Knuutila, Conventional osteosarcoma, in: Pathology and Genetics of Tumours of Soft Tissue and Bon, C.D.M. Fletcher, K.K. Unni and F. Mertens, eds, Lyon: IARCPress, 2002.

2) S.S. Bielack, B. Kempf-Bielack, G. Delling et al., Prognostic factors in highgrade osteosarcoma of the extremities or trunk: an analysis of 1,702 patients treated on neoadjuvant cooperative osteosarcoma study group protocols, J Clin Oncol 20 (2002), 776-790.

3) J.C. Clark, C.R. Dass and P.F. Choong, A review of clinical and molecular prognostic factors in osteosarcoma, J Cancer Res Clin Oncol 134 (2008), 281297.

4) A. Patiño-García, M. Zalacain, C. Folio et al., Profiling of chemonaive osteosarcoma and paired-normal cells identifies EBF2 as a mediator of osteoprotegerin inhibition to tumor necrosis factor-related apoptosis-inducing ligand-induced apoptosis, Clin Cancer Res 15 (2009), 5082-5091.

5) A.G. Van Rossum, W.H. Moolenaar and E. Schuuring, Cortactin affects cell migration by regulating intercellular adhesion and cell spreading, Exp Cell Res 312 (2006), 1658-1670.

6) E. Schuuring, The involvement of the chromosome $11 \mathrm{q} 13$ region in human malignancies: cyclin D1 and EMS1 are two new candidate oncogenes-a review, Gene 159 (1995), 83-96.

7) J. Atiye, M. Wolf, S. Kaur et al., Gene amplifications in osteosarcoma - CGH microarray analysis, Genes Chromosomes Cancer 42 (2005), 158-163.

8) F. Lecanda, P.M. Warlow, S. Sheikh et al., Connexin 43 deficiency causes delayed ossification, craniofacial abnormalities, and osteoblast dysfunction, $\mathrm{J}$ Cell Biol 151 (2000), 931-944.

9) T. Ezponda, M.J. Pajares, J. Agorreta et al, The oncoprotein SF2/ASF promotes non-small cell lung cancer survival by enhancing survivin expression, Clin Cancer Res 16 (2010), 4113-4125.

10) L.I. Cosen-Binker and A. Kapus, Cortactin: The Gray Eminence of the Cytoskeleton, Physiology 21 (2006), 352-361.

11) L. Buday and J.J. Downward, Roles of cortactin in tumor pathogenesis, Biochim Biophys Acta 1775 (2007), 263-273.

12) K.C. Kirkbride, B.H. Sung, S. Sinha and A.M. Weaver, Cortactin: A multifunctional regulator of cellular invasiveness, Cell Adh Migr 5 (2011), 187198.

13) C.C. Lau, C.P. Harris, X.Y. Lu et al., Frequent amplification and rearrangement of chromosomal bands 6p12-p21 and $17 \mathrm{p} 11.2$ in osteosarcoma, Genes Chromosomes Cancer 39 (2004), 11-21.

14) B.L. Rothschild, A.H. Shim, A.G. Ammer et al., Cortactin overexpression regulates actin-related protein $2 / 3$ complex activity, motility, and invasion in carcinomas with chromosome 11q13 amplification, Cancer Res 66 (2006), 8017-8025.

15) A. Maitra, H. Roberts, A.G. Weinberg and J. Geradts, Loss of p16(INK4a) expression correlates with decreased survival in pediatric osteosarcomas, Int J Cancer 95 (2001), 34-38.

16) L. Molendini, M.S. Benassi, G. Magagnoli et al., Prognostic significance of cyclin expression in human osteosarcoma, Int J Oncol 12 (1998), 1007-1011. 
17) M.S. Benassi, L. Molendini, G. Gamberi et al., Altered G1 phase regulation in osteosarcoma, Int J Cancer 74 (1997), 518-522.

18) A.M. Weaver, Cortactin in tumor invasiveness, Cancer Lett 265 (2008), 157166.

19) Y. Yamaguchi and J. Condeelis, Regulation of the actin cytoskeleton in cancer cell migration and invasion, Biochim Biophys Acta 1773 (2007), 642-652. 
Table 1. Clinical and histopathologic features of the osteosarcoma patients

\begin{tabular}{|c|c|c|c|c|c|c|c|}
\hline Patient & Histology & Age (yr) & Gender & Location & Necrosis & Follow-up & $\begin{array}{c}\text { Suvival } \\
\text { (mo) }\end{array}$ \\
\hline 473 & Fibroblastic & 15.08 & M & Tibia & $95 \%$ & Remission & 96 \\
\hline 475 & Osteoblastic & 23.5 & $\mathrm{~F}$ & Femur & $100 \%$ & Remission & 96 \\
\hline 486 & Osteochondroblastic & 24 & M & Tibia & ${ }^{1} \mathrm{NA}$ & Died of disease & 14 \\
\hline 491 & Osteochondroblastic & 16.6 & M & Femur & $90 \%$ & Died of disease & 84 \\
\hline 500 & Osteoblastic & 11 & M & Femur & $>90 \%$ & Remission & 90 \\
\hline 524 & Osteochondroblastic & 11 & $\mathrm{~F}$ & Femur & $<10 \%$ & Remission & 90 \\
\hline 531 & Osteochondroblastic & 22 & $\mathrm{~F}$ & Multifocal & $85 \%$ & Died of disease & 27 \\
\hline 554 & Telangiectasic & 16 & M & Femur & $90 \%$ & Remission & 108 \\
\hline 588 & Osteochondroblastic & 16 & M & Tibia & $70 \%$ & Died of disease & 46 \\
\hline 595 & Osteoblastic & 16.66 & $\mathrm{~F}$ & Femur & $90 \%$ & Died of disease & 7 \\
\hline 598 & Osteoblastic & 14 & $\mathrm{~F}$ & Femur & NA & Died of disease & 7 \\
\hline 605 & Osteoblastic & 27.75 & $\mathrm{~F}$ & Sacro-iliac & NA & Died of disease & 3 \\
\hline 628 & Osteoblastic & 13.25 & M & Femur & $95 \%$ & Remission & 47 \\
\hline 631 & Osteoblastic & 12.16 & M & Femur & $100 \%$ & Remission & 45 \\
\hline 636 & Osteoblastic & 15 & M & Femur & $99 \%$ & Remission & 45 \\
\hline 648 & Osteoblastic & 15.66 & $\mathrm{~F}$ & Tibia & $100 \%$ & Remission & 52 \\
\hline 652 & Osteoblastic & 10.75 & M & Tibia & $100 \%$ & Remission & 36 \\
\hline 661 & Osteoblastic & 14.58 & $\mathrm{~F}$ & Femur & $95 \%$ & Died of disease & 7 \\
\hline 664 & Chondroblastic & 12 & M & Femur & $>90 \%$ & Remission & 20 \\
\hline 669 & Osteoblastic & 16.5 & M & Femur & $70 \%$ & Died of disease & 13 \\
\hline
\end{tabular}

${ }^{1} \mathrm{NA}$ : not available. 

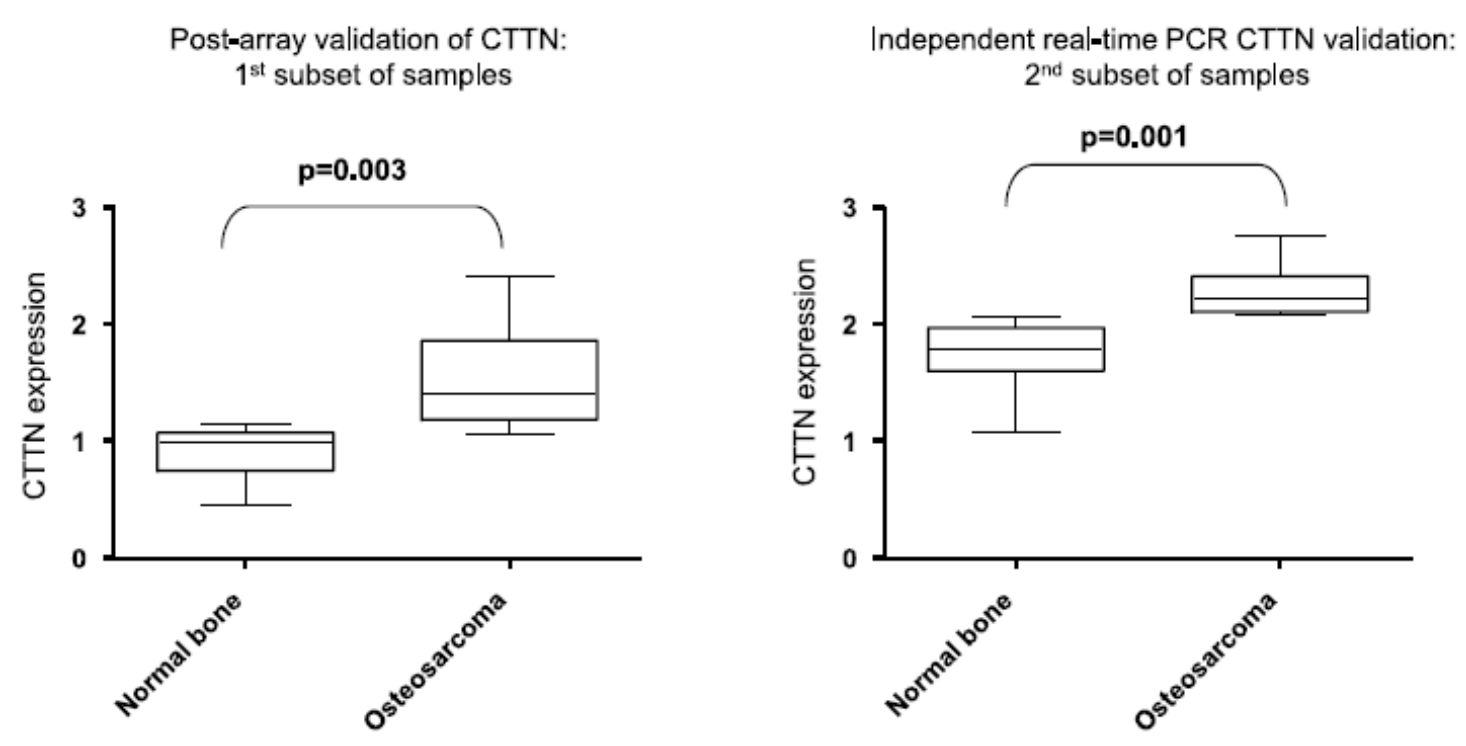

Figure 1. Representation of CTTN relative expression in pediatric osteosarcoma tissues and normal bones by real-time PCR.

\section{CTTN IHC}

a) Primary osteosarcoma

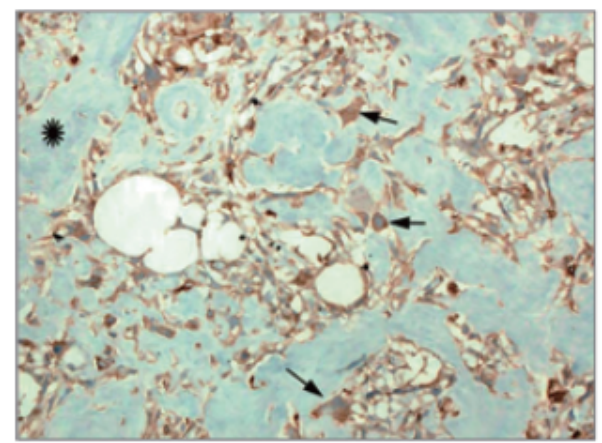

c) Normal bone

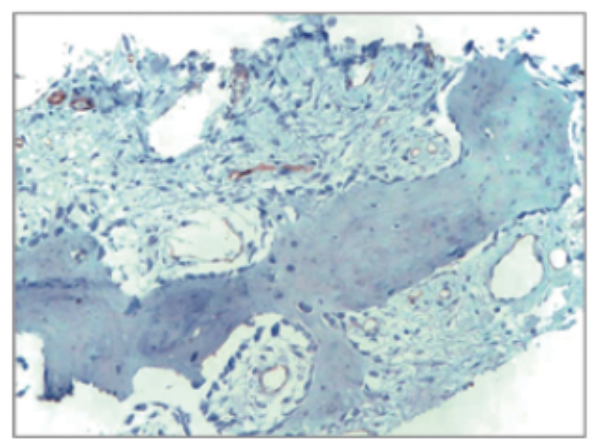

b) Osteosarcoma lung metastasis

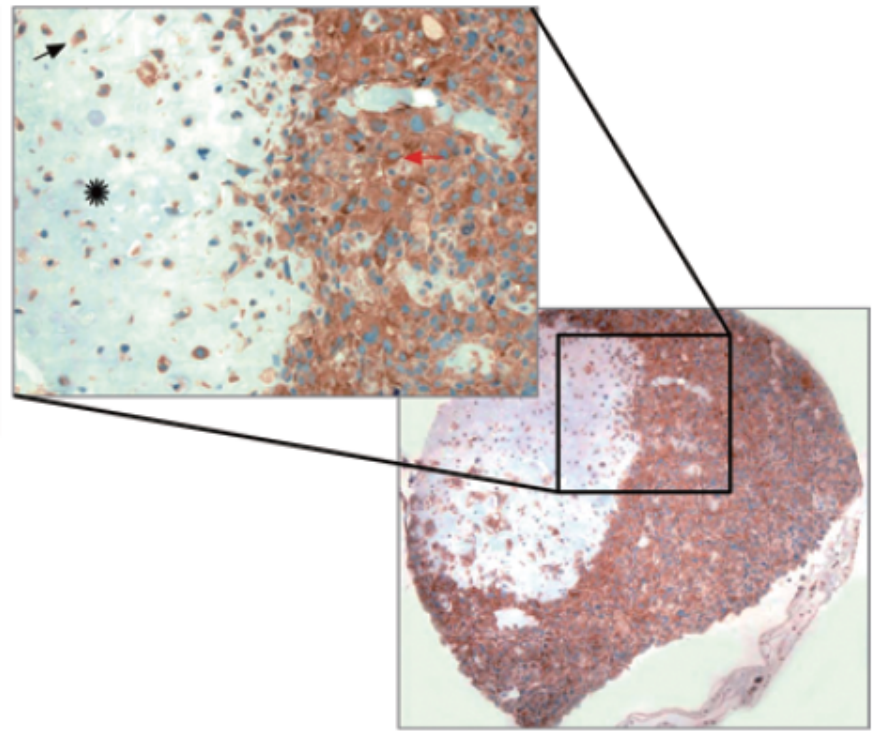

Figure 2. Cytoplasmic staining of CTTN in a primary tumor (a) and a lung metastasis (b) compared to a normal bone (c), showing the typical different intensity of staining. The asterisk marks tumoral osteoid; the arrows indicate examples of stained tumoral osteoblasts. (IHC, 200x). 


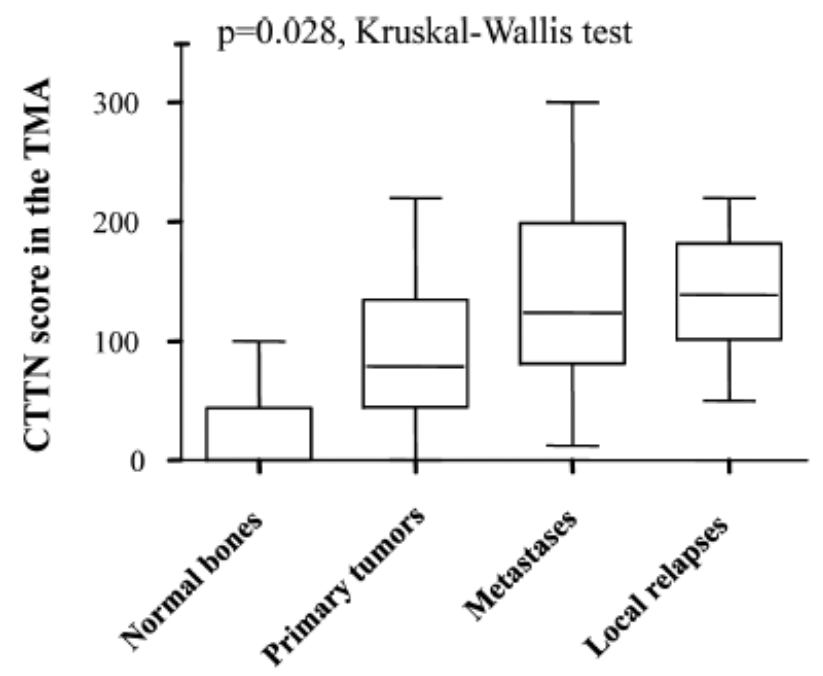

Post-hoc Tukey HSD test comparisons:
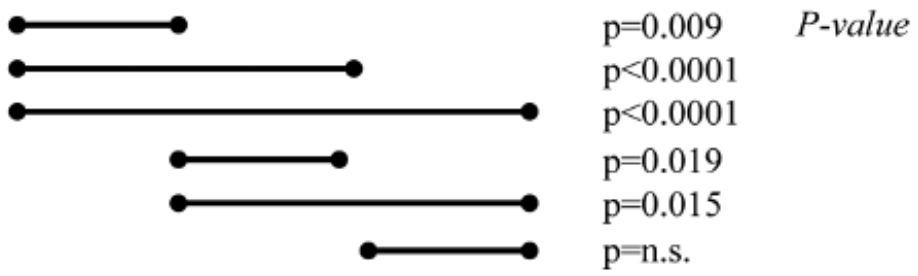

Figure 3. Box plot representing the median CTTN score for the different osteosarcoma stages analyzed. On the $\mathrm{Y}$ axis an arbitrary scale represents the IHC score (see Materials and Methods section).

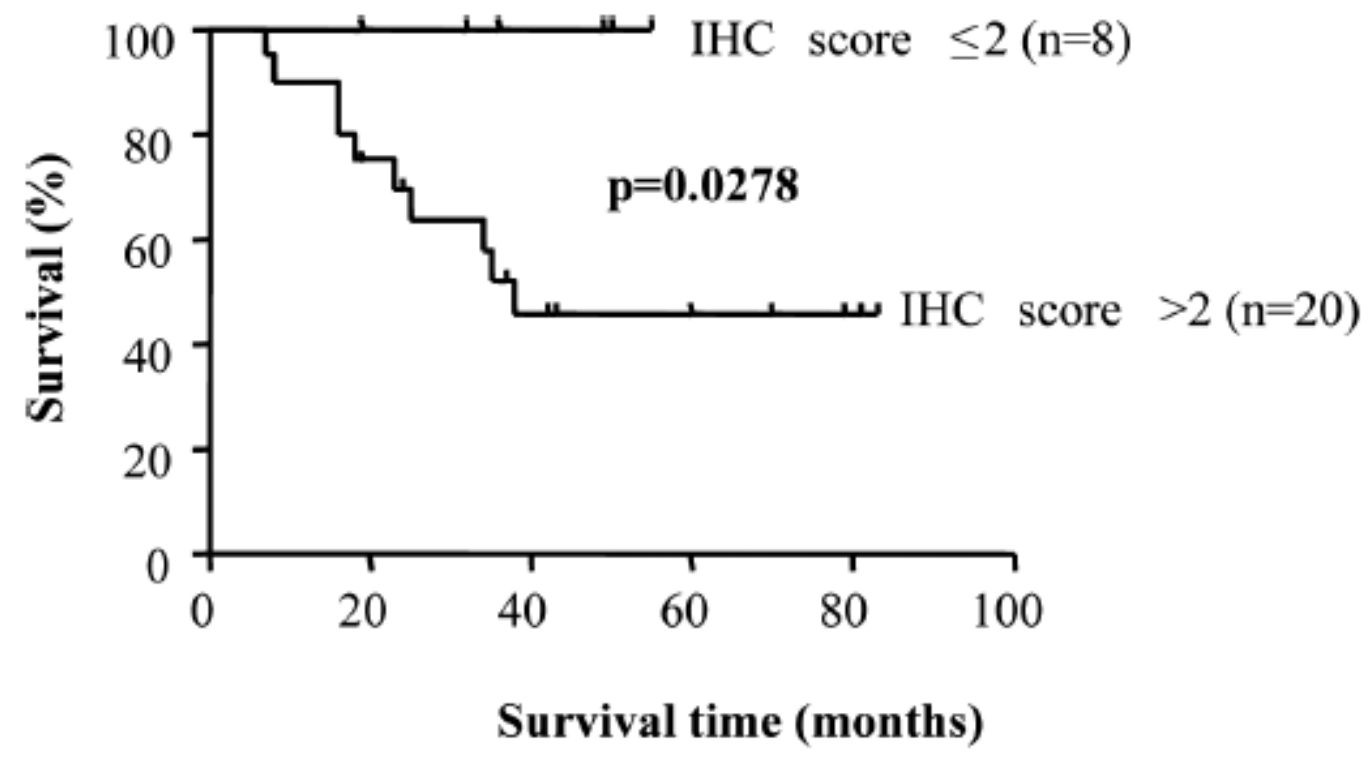

Figure 4. Kaplan-Meier survival curves for primary osteosarcomas according to IHC cortactin expression. 